\title{
Arrest of Domain Coarsening via Anti-periodic Regimes in Delay Systems
}

\author{
J. Javaloyes, ${ }^{1}$ T. Ackemann, ${ }^{2}$ and A. Hurtado ${ }^{3}$ \\ ${ }^{1}$ Departament de Fisica, Universitat de les Illes Baleares, \\ C/ Valldemossa km 7.5, 07122 Mallorca, Spain. \\ ${ }^{2}$ SUPA and Department of Physics, University of Strathclyde, \\ John Anderson Building, $10^{7}$ Rottenrow, Glasgow G4 0NG, UK. \\ ${ }^{3}$ Institute of Photonics, SUPA and Department of Physics, University of Strathclyde, \\ Technology and Innovation Centre, 99 George Street, Glasgow G1 1RD, UK
}

\begin{abstract}
Motionless domains walls representing heteroclinic temporal or spatial orbits typically exist only for very specific parameters. This report introduces a novel mechanism for stabilizing temporal domain walls away from the Maxwell point opening up new possibilities to encode information in dynamical systems. It is based on anti-periodic regimes in a delayed system close to a bistable situation, leading to a cancellation of the average drift velocity. The results are demonstrated in a normal form model and experimentally in a laser with optical injection and delayed feedback.
\end{abstract}

PACS numbers: 42.65.Sf, 02.30.Ks, 05.45.-a, 89.75.Fb

Spatially extended nonlinear systems often admit multiple coexisting stable states, and the dynamical properties of the fronts connecting them are fundamental in the understanding of pattern formation and localized structures (LS). These dissipative objects [1-11] occur in many natural and laboratory systems and are characterized by a correlation range much shorter than the size of the system, making them individually addressable. Localized structures due to a front between a homogeneous and a cellular state are intrinsically stable [12-14], as the pattern oscillations stabilize the position of the front against small perturbations. In contrast, fronts between homogeneous states are typically stable only for very specific parameters and lead otherwise to a coarsening.

In this letter, we discuss a robust mechanism for the stabilization of fronts between homogeneous states based on a generic property of time delayed systems having no equivalent in spatially extended systems. We show that structures occurring within a delay time can be stabilized by a temporal inversion after each time delay, thus allowing the stabilization of self-localized temporal domains in temporal slices modulo twice the delay. The case of equispaced domain walls reduces to the so-called square-waves $[15,16]$. This has not only relevance for the fundamentals of the dynamics of delayed system, but also meets recent interest in temporal LS realized in photonics [17-23]. Due to their intrinsically fast dynamics, the possibility to use LS as bits for information processing was addressed early in nonlinear optics [24-26]. Interesting results were achieved for spatial solitons in semiconductor microcavities [27-29], although it turned out that spatial disorder limits parallelism and control [30, 31], motivating further studies into temporal LS.

As indicated, in the simple case of a unidimensional bistable system with a single dynamical variable $\psi(x, t)$, the stable coexistence between two homogeneous phases is merely achieved for a single parameters' value, the socalled Maxwell point. Here a domain wall separating the two states would be motionless. Yet, such a regime possesses little experimental significance since any deviation of the control parameter or any symmetry breaking effect implies that one of the two bistable phases will eventually invade the other in a way reminiscent of nucleation bubbles in first order phase transitions.

In recent years, building on the strong analogies between spatial and delayed systems [32-34], a similar symmetry breaking induced coarsening dynamics was shown to occur in delayed bistable systems [35, 36]. The ability to control the motion of these walls would have significant implications as for instance to encode and process information with a fast nonlinear delayed system. Motivated by this idea, the pinning of domain walls was recently demonstrated via an external temporal modulation [37]. Departing from the analogies with spatial systems, we demonstrate in contrast a stabilization mechanism based not upon a fast active modulation, but upon a slow, self-induced dynamics. We envision the use of a lesser known property of delayed systems: their ability to generate anti-periodic output, i.e. temporal traces getting inverted after each time delay $\tau$, thereby inducing an effective periodicity $2 \tau$.

While this effect was studied theoretically in $[15,16$, 38 ] and square-waves were demonstrated experimentally in several optical [39, 40] and opto-electronic systems [41], we demonstrate in this letter how this generic property of anti-periodicity can be harnessed to create robust motionless domain walls and prevent the coarsening phenomenon. This allows to store information even far from the Maxwell point and/or in the absence of bistability. Such an idea has no equivalent in bona fide spatial systems since the anti-periodicity would actually correspond to a space defined over a Möbius strip. We evidence experimentally and theoretically stable domains in an injected semiconductor laser with delayed feedback, that are insensitive to symmetry breaking and exist beyond the bistability.

We base our analysis on the normal form of the imperfect pitchfork bifurcation modified by the inclusion of a 
linear delayed contribution

$$
\varepsilon \frac{d \Psi}{d t}=\mu \Psi+\beta \Psi^{2}-\Psi^{3}+\eta \Psi(t-1) .
$$

In Eq. (1), the parameter $\mu$ controls the bistability, $\beta$ represents the symmetry breaking and $\eta$ is the amplitude of the time delayed feedback. The temporal scale is normalized by the time delay $\tau$. We study the limit of long delays $\tau \rightarrow \infty$, and define a smallness parameter $\varepsilon=1 / \tau$ making apparent the singular nature of Eq. (1). Delay Differential Equations (DDEs) possess in the long delay limit an eigenvalue spectrum that can be divided in two parts, see for instance [42] and references therein. A quasi-continuous branch stems from the influence of the delayed contribution while a discrete spectrum is generated by the instantaneous linear terms. We are interested in the regimes where a portion of the quasi-continuous branch may become unstable giving rise to smooth dynamics. We set $\mu<0$ ensuring the stability of the discrete spectrum. When $\varepsilon \rightarrow 0$, the left hand side of Eq. (1) can be assumed, as a first approximation, to vanish. Such an approach is enlightening as one finds a functional mapping governing the evolution of the small deviations from the trivial solution $\Psi=0$ as

$$
\Psi(t)=-\kappa \Psi(t-1)
$$

with $\kappa=\eta \mu^{-1}$. Due to the infinite dimensionality of DDEs, an initial condition must be given as a function defined over an interval equal to the delay. If $|\kappa|<1$, any initial condition slowly decays from one round-trip to the next and the steady state $\Psi(t)=0$ is asymptotically stable. Yet, for $|\kappa|>1$, the trivial solution may bifurcate via two widely different scenarios. If $\kappa<-1$, the temporal profile evolves regularly from one round-trip to the next while for $\kappa>1$, the profile gets inverted at each round-trip signaling the onset of a period-2 (P2) regime.

The slowly evolving dynamics of the temporal profile can be better understood and visualized via the spatiotemporal equivalence between delayed and spatially extended systems [16, 32-34, 42]. We define the deviation from the two bifurcation points $\mu=\kappa \eta$ with $\kappa= \pm 1$, as $\mu=a+\varepsilon^{2} a_{1}$ and $\eta=a \kappa+b_{1} \varepsilon^{2}$ and, as detailed in [42], inserting a multiple time scales expansion in Eq. (1) for both the temporal derivative and the delayed term, and defining $\Psi=\varepsilon \psi+\mathcal{O}\left(\varepsilon^{2}\right)$, one obtains the following equivalent partial differential equation (PDE) as a third order solvability condition

$$
\frac{\partial \psi}{d n}=p \psi+\frac{\beta}{a} \psi^{2}+\frac{\psi^{3}}{a}+\frac{1}{2 a^{2}} \frac{\partial^{2} \psi}{\partial x^{2}},
$$

with $p=-\left(a_{1}-\kappa b_{1}\right) / a$. Although strictly valid in the vicinity of the bifurcation points, such normal forms are known to have a wider domain of validity, see the discussion before Eq. (8a) in [16]. In Eq. (3), the spatial coordinate $(x)$ must be understood as a local time coordinate within the round-trip while the slow time $(n)$ represents the evolution of the temporal profile from one round-trip to the next. Besides, we factored out a drift
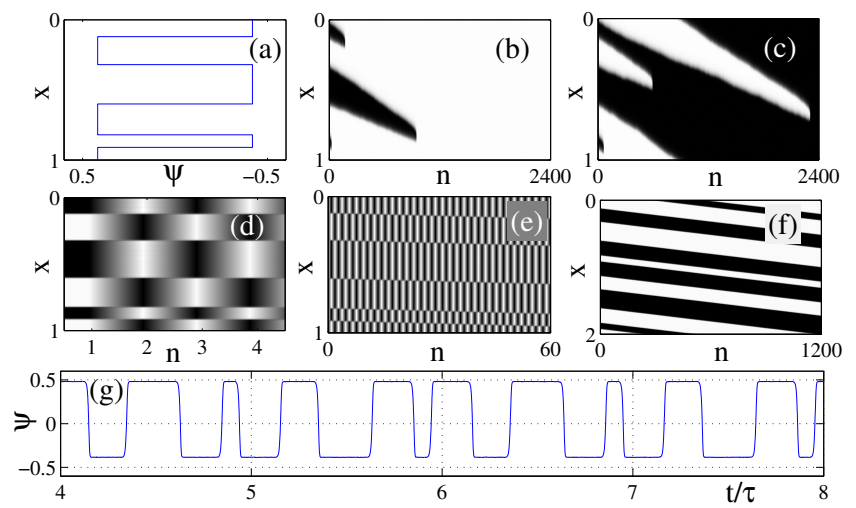

Figure 1. (color online). Illustration of dynamics in space-time diagrams. These space-time diagrams are folded over a time $T=$ $\tau+|\eta|^{-1}$. a) Temporal pattern imposed as an initial condition. The $\mathrm{P} 1$ regime where $\kappa=-1$ leads to the coarsening dynamics of the temporal time trace depicted in b) and c) towards the high and low states when $\beta=-10^{-2} \mathrm{~b}$ ) and $\beta=10^{-2} \mathrm{c}$ ), respectively. Other parameters are $\tau=10^{3}, \eta=5 \times 10^{-2}$ and $\mu=2.5 \times 10^{-2}$, or equivalently, $a=-5 \times 10^{-2}, b_{1}=0$ and $a_{1}=7.5 \times 10^{4}$. The P2 regime where $\kappa=1$, induces the inversion of the initial condition at each round-trip as exemplified by the checkerboard pattern in d). It does not coarsen over longer time scales even for very large values of the asymmetry $\beta=0.1$. The time trace is folded over times $T$ in e) and also $2 T$ in f) for clarity. Parameters are $\eta=$ -0.2 and $\mu=-2.5 \times 10^{-2}$, or equivalently $a=-0.2, b_{1}=0$ and $a_{1}=17.25 \times 10^{4}$. The temporal profile is represented in $\mathrm{g}$ ). Notice the large values of $a_{1}$ signaling that Eq. (3) remains qualitatively valid far from the bifurcation points.

velocity defined as $v=a^{-1}$ as in [23,33], representing the small deviation of the period with respect the time delay. Equation (3) must be complemented by a boundary condition that reads

$$
\psi(x+1, n)=-\kappa \psi(x, n)
$$

and defines, as in Eq. (2), whether or not the solution gets inverted from one round-trip to the next. For $\beta=0$ and $a<0$, the solutions of the heteroclinic orbits of Eq. (3) read $K_{ \pm}(x)= \pm \sqrt{|a| p} \tanh (|a| \sqrt{p} x)$ where the \pm stands for the upward and downward domains walls. In deriving Eq. (1) we assumed that the symmetry breaking term $\beta$ scales as $\varepsilon$ to enter as a perturbation of the solvability condition. Yet, although small, $\beta$ has a deep impact upon the dynamics.

We depict in Fig. 1a) an initial condition for Eq. (1) composed of an arbitrary succession of domains with values corresponding to the plateaus of the $\mathrm{P} 1$ or of the P2 regimes. In the $\mathrm{P} 1$ case $(\kappa=-1)$ as visible in Fig. $1 \mathrm{~b}, \mathrm{c})$, this multi-plateau pattern relaxes in a finite time to the upper (resp. lower) value when $\beta<0$ (resp. $\beta>0$ ). Such a coarsening scenario is very general and it is preserved quite far from the bifurcation condition $\mu=-\eta$, and even when the discrete spectrum is also unstable, i.e. with $\mu>0$. A multi-domain solution that verifies Eqs. $(3,4)$ can be written as

$$
\psi(x, n)=K_{+}\left(x-x_{0}^{+}\right)+K_{-}\left(x-x_{1}^{-}\right)
$$




$$
+\cdots+K_{-}\left(x-x_{n}^{-}\right)-\sqrt{|a| p}
$$

with $\left\{x_{n}^{ \pm}\right\}$the ordered collection of the coordinates of the upward and downward kinks. For $|\beta| \ll 1$, a variational approach is justified [43], and we insert an ansatz as $\psi(x, s)=K_{ \pm}\left[x-x_{ \pm}(s)\right]$. By multiplying Eq. (3) by $\partial_{x} K_{ \pm}$and integrating over the spatial coordinate, we find the effective equation for the motion of an isolated wall as

$$
\frac{d x_{i}^{ \pm}}{d n}=\mp \frac{\beta}{2 a \sqrt{|a|}}
$$

demonstrating that the symmetry breaking term $\beta$ induces a splitting of the velocity of domain walls of opposed "charge", that will eventually collide. Notice that the value of the drift can also be found searching for heteroclinic solutions of Eq. (1) as in [35]. Both methods neglect the short range, exponentially decreasing interactions between nearby walls that is known to be attractive $[16,38,43]$. This can leads for $\beta<0$ to a steady state, that is however unstable and cannot prevent the eventual collision and coarsening. Hence, one may conclude that it is impossible to store information in a scalar delayed system as any symmetry breaking nonlinearity leads to a coarsening of the information.

We now demonstrate how a completely different regime can be obtained exploiting the anti-periodic solutions of Eq. (1) achieved by simply setting $\eta<0$ to access the regime where $\kappa=1$. We stress that here since $\mu<0$, bistability is lost and the only steady solution is $\Psi=0$. Surprisingly, stable domains with well defined plateaus can be obtained even in this regime, although the time trace gets inverted every round-trip, thereby inducing a periodicity close to twice the delay value $2 \tau$, see Fig. 1d$\mathrm{g})$. Although the solution inversion is interesting in its own right, such a behavior can be deduced intuitively from the singular mapping Eq. (2) setting $\kappa=1$. The striking result is that the domain walls remain motionless, even for large $\beta$ values, see Fig. 1d) and Fig. 1e) where the checkerboard pattern visually disappears leaving only visible the transitions. We verified this robustness with other kind of symmetry breaking terms e.g. $\gamma_{m} \Psi^{m}$ with $m=0$ and $m=4$. One can still construct approximate analytic solutions of Eq. (3) where an upward or a downward kink must be complemented by an opposed kink at a spatial distance $x=1$ in order to fulfill the anti-periodic boundary condition Eq. (4). A similar variational approach allows us to find the effective equation of motion of an isolated wall,

$$
\frac{d x_{i}}{d n} \sim \beta \int_{0}^{2}\left(\partial_{x} K_{+}+\partial_{x} K_{-}\right)\left(K_{+}+K_{-}\right)^{2} d x=0 .
$$

In other words, by inverting at each round-trip, the walls experience opposed drift velocities that cancel out explaining why similar results can be found for other kind of symmetry breaking nonlinearities. It was also demonstrated in [16] that the quadratic term cancels out of
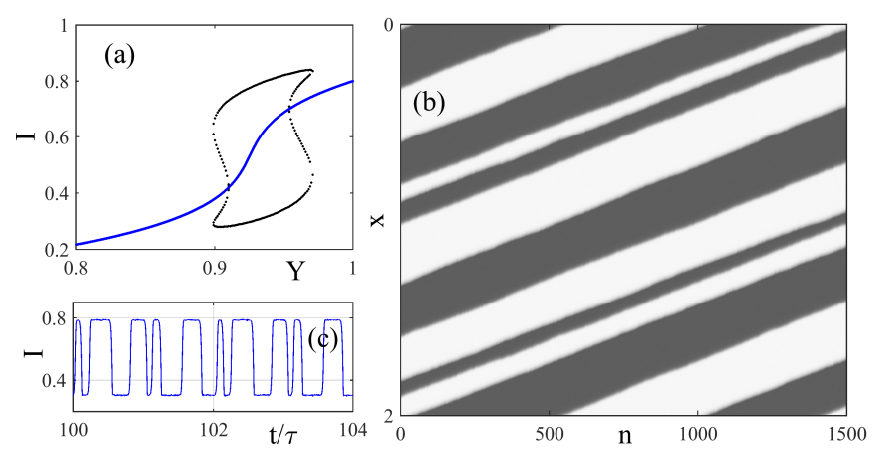

Figure 2. (color online): a) Non linear output intensity $I=|E|^{2}$ as a function of the injected field $Y$ in blue. The black dots represents the amplitude of the upper and lower plateau in the P2 regime. The space-time diagram of the intensity in panel b) is folded with a period $T=2 \tau+0.1$ and shows the stability of the domain walls, even in the presence of noise, see also panel c). Parameters are $\alpha=2, J=-0.1, \Delta=2.3, \tilde{\eta}=0.1$ and $\Omega=\pi$.

Eq. (3) even if it is large, see Eqs. (A.17-18) in the appendix A of [16] for details. Higher order nonlinearities in Eq. (1) potentially as large as $\gamma_{m} \sim \varepsilon^{3-m}$ may enters Eq. (3), but would cancel out similarly in Eq. (7). We conclude that P2 delayed systems are robust candidates for information storing as they are impervious to most experimental imperfections leading to a coarsening.

We study the case of an injected semiconductor laser below threshold subject to optical feedback, as domain stabilization in telecommunication lasers is very interesting also from an applicative point of view. Close, yet below, threshold and in the limit of weak optical feedback, weak optical injection, small detuning and large delay, one can reduce the standard single-mode rate equations of the semiconductor laser to the following delayed Ginzburg-Landau equation

$\dot{E}=(1+i \alpha)\left(J-|E|^{2}\right) E+i \Delta E+Y+\tilde{\eta} e^{-i \Omega} E(t-\tau)(8)$

see for instance [23] for details. Here, $J$ denotes the deviation from threshold of the bias current. The detuning between the frequency of the injection field $\omega_{Y}$ of amplitude $Y$ (chosen real by definiteness) and the free running frequency of the laser scaled by the photon lifetime is $\Delta$, whilst $\tilde{\eta}$ and $\Omega=\omega_{Y} \tau$ are the amplitude and phase of the delayed optical feedback, respectively. At steady state $(\dot{E}=0)$, the output power $|E|^{2}$ as a function of $Y$ can present a bistable S-shape response for some parameters. We work close to the onset of bistability where the transition from low to high power has a sigmoid shape, see the blue line in Fig. 2a).

Assuming that a P2 solution evolves between two plateaus whose values are $E_{ \pm}$, it yields a system of equations whose solution is represented by the black dots in Fig. 2a,b). Such a diagram suggests that these P2 regimes are nascent from two saddle-node bifurcations of limit cycles and are also connected to the stationary solution (blue line) by two Andronov-Hopf bifurcations. 
(a)

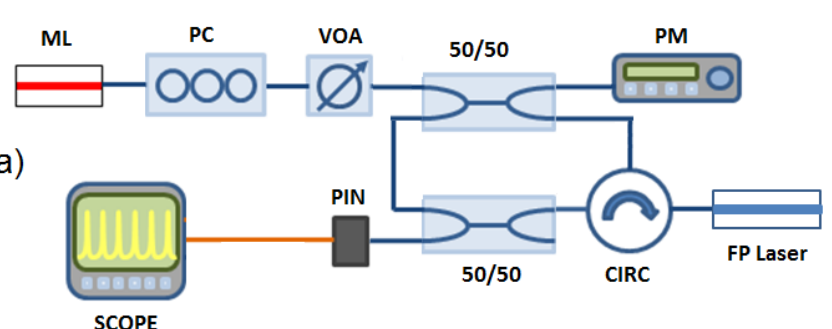

(b)

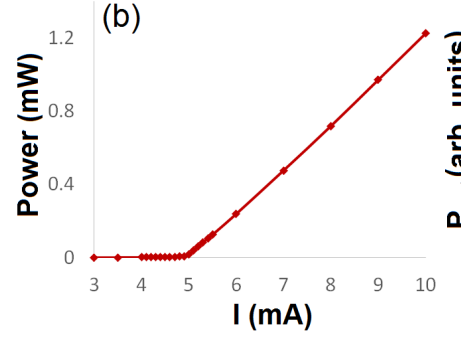

(c)

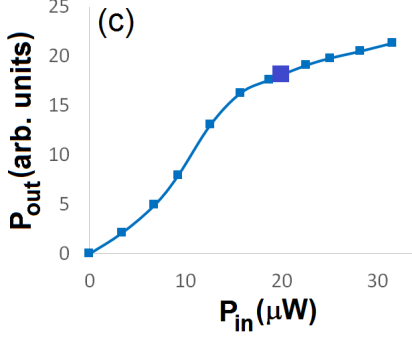

Figure 3. (color online) (a) Experimental Setup. Legend: ML: Master Laser; PC: Polarization Controller; VOA: Variable Optical Attenuator; PM: Power Meter; FP: Fabry-Perot; CIRC: Circulator; PIN: $12 \mathrm{GHz}$ Photodetector; SCOPE: $13 \mathrm{GHz}$ Oscilloscope. (b) LIcurve of the SL at $T=293 \mathrm{~K}$. (c) I/O power relationship of the SL under external injection with $\Delta f=-10 \mathrm{GHz}$ and $I=4.85 \mathrm{~mA}$.

However these bifurcations are subcritical meaning that the equivalent PDE would certainly take the form of a subcritical cubic-quintic Ginzburg-Landau equation. Notice that for dimensional systems with a higher number of variables, as in Eq. (8), additional instabilities like the Eckhaus mechanism [44] may arise. Yet, Eq. (8) can be reduced to a single scalar delayed equation for the phase in the limit of weak feedback and injection using averaging methods [45]. We show indeed in Fig. 2b,c) that this system is capable storing patterns of domain walls.

The experimental setup is represented in Fig. 3. A $1310 \mathrm{~nm}$ Fabry-Perot Laser (Slave Laser, SL) as used in telecommunication systems is subject simultaneously to external optical injection and delayed feedback after a round-trip time $\tau=65.4 \mathrm{~ns}$. The optically injected signal was generated with a tunable laser (Master Laser, ML). A polarization controller and a variable optical attenuator were respectively included after the ML to control its polarization state and optical power level. Figure $3 \mathrm{~b}$ ) plots the light-current (LI) curve of the solitary SL, showing a threshold current of $I_{t h}=4.92 \mathrm{~mA}$. Figure 3c) depicts the SL's input/output power relationship when subject solely to external injection. The device was biased below threshold with a current of $I=4.85 \mathrm{~mA}$ (i.e. $\left.I=0.985 I_{t h}\right)$ and an initial frequency detuning $\left(\Delta f=f_{i n j}-f_{F P}\right)$ equal to $-10 \mathrm{GHz}$ was set between the frequencies of the injected signal and the resonance frequency of one of the SL modes. Figure 3c) illustrates the achievement of a gradual nonlinear switching response as the injection strength is increased from 0 to $31.5 \mu \mathrm{W}$ in agreement with the results of Fig. 2a).

The SL was subsequently subjected to simultaneous optical injection from the ML (input power $P_{i n j}=$

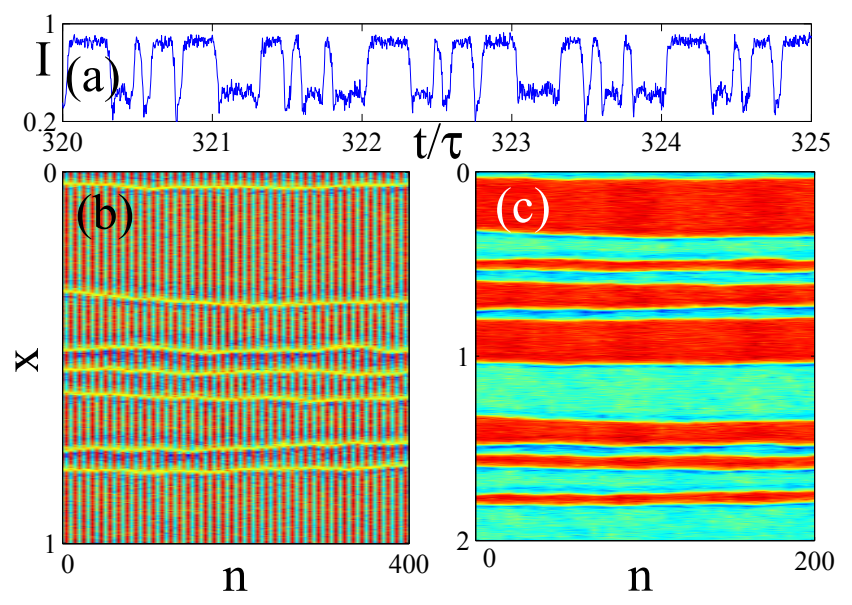

Figure 4. (color online). Temporal trace of the laser intensity a) and space-time diagram with folding parameter $\tau$ b) and $2 \tau \mathrm{c}$ ).

$20 \mu \mathrm{W})$ and delayed feedback. In this situation the system was operated in the sigmoid range as indicated by the blue square in Fig. 3c). The phase of the feedback can be changed by a small detuning of the injection field frequency. The large values of the time delay allows considering these two parameters independent.

Figure $4 \mathrm{a})$ plots time trace over 5 round-trips ( $327 \mathrm{~ns}$ ). Figure 4a) shows that a temporal pattern which is inverted every round-trip is obtained at the device's output, in agreement with the predictions of Fig. 1g) and Fig. 2c). The equivalent space-time diagrams for the time series of Fig. 4a) when the folding parameter is set approximately to $\tau$ and $2 \tau$ are plotted respectively in Fig. 4b) and Fig. 4c) over a time window of 400 roundtrips (i.e. $\sim 26 \mu \mathrm{s}$ ). The figures $4 \mathrm{~b}-\mathrm{c}$ ) demonstrate the experimental achievement of anti-periodic dynamical regimes since peaks-troughs alternate every round-trip, see Fig. 4b). More importantly, the results also demonstrate the formation of stable domains of arbitrary size. The existence of various noise sources in the system induces a slow drift of the walls as seen in Fig. 4c), but not a coarsening if the walls remain at a sufficient large distance, in good agreement with theory.

Noteworthy, we observed the nucleation of domain wall pairs after the arrival of an electrical perturbation into the system as shown in Fig. 5a) at $n=128$. Additionally, a small change of the injection parameters allows to pass into the weakly bistable regime and to observe in Fig. 5b) the coarsening mechanism of the P1 solutions, as in [35].

In conclusion, we presented in this manuscript how a general property of delayed systems can be harnessed to prevent the domain coarsening in symmetry broken delayed systems thereby allowing the storing of information. Such anti-periodicity has no equivalent in real spatially extended systems. We evidence the existence of stable domains in the coherent output of a semiconductor laser with optical feedback. These results offer exciting prospects for the controllable encoding of information. 


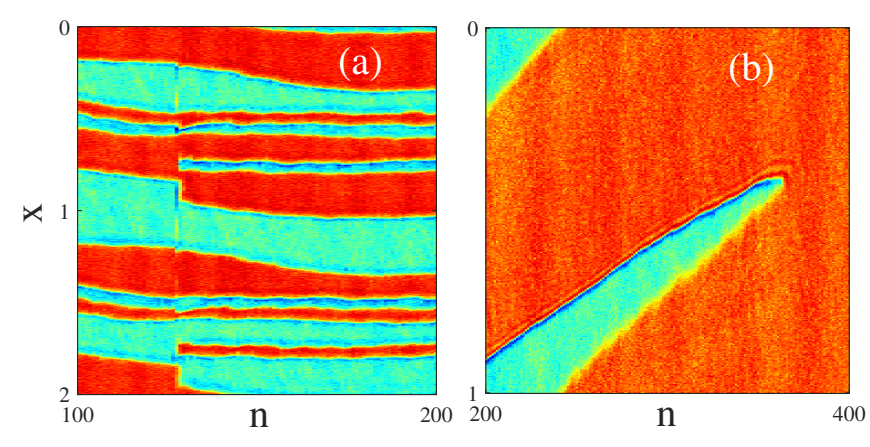

Figure 5. (color online). Same as Fig. 4. A new pair of domain wall is nucleated at $n=128$ in panel a) and remain stable while for different parameters bistable phase coarsening occurs.

\section{ACKNOWLEDGMENTS}

J.J. acknowledges useful discussion with S. Balle as well as financial support from Ramón y Cajal program, and project RANGER (TEC2012-38864-C03-01). A.H. thanks Prof. A. Kemp for lending the oscilloscope used in the experiments and financial support from the Stratchlyde Chancellor's Fellowships Programme: Starter Grant (Ref 12431DP2425AOOO), Institute of Photonics, University of Strathclyde
[1] S. Fauve and O. Thual. Solitary waves generated by subcritical instabilities in dissipative systems. Phys. Rev. Lett., 64:282-284, Jan 1990.

[2] N. Akhmediev and A. Ankiewicz. Dissipative Solitons, volume 661 of Lecture Notes in Physics. Springer Berlin Heidelberg, 2005.

[3] N. Akhmediev and A. Ankiewicz. Dissipative Solitons: From Optics to Biology and Medicine Series, volume 751 of Lecture Notes in Physics. Springer Berlin Heidelberg, 2008.

[4] P. B. Umbanhowar, F. Melo, and H. L. Swinney. Localized excitations in a vertically vibrated granular layer. Nature, (382):793 - 796, 1996.

[5] Yuri A. Astrov and H.G. Purwins. Plasma spots in a gas discharge system: birth, scattering and formation of molecules. Physics Letters A, 283(5-6):349 - 354, 2001.

[6] Yu. Astrov, E. Ammelt, and H.-G. Purwins. Experimental evidence for zigzag instability of solitary stripes in a gas discharge system. Phys. Rev. Lett., 78:3129-3132, 1997.

[7] F. J. Niedernostheide, M. Arps, R. Dohmen, H. Willebrand, and H. G. Purwins. Spatial and spatio-temporal patterns in pnpn semiconductor devices. physica status solidi (b), 172(1):249 - 266, 1992.

[8] Kyoung-Jin Lee, William D. McCormick, John Pearson, and Harry L. Swinney. Experimental observation of selfreplicating spots in a reaction-diffusion system. Nature, 369:215-218, 1994.

[9] J. Wu, R. Keolian, and I. Rudnick. Observation of a nonpropagating hydrodynamic soliton. Phys. Rev. Lett., 52:1421-1424, Apr 1984.

[10] E. Moses, J. Fineberg, and V. Steinberg. Multistability and confined traveling-wave patterns in a convecting binary mixture. Phys. Rev. A, 35:2757-2760, Mar 1987.

[11] L.A. Lugiato. Introduction to the feature section on cavity solitons: An overview. Quantum Electronics, IEEE Journal of, 39(2):193-196, 2003.

[12] Y. Pomeau. Front motion, metastability and subcritical bifurcations in hydrodynamics. Physica D, 23:3-11, 1986.

[13] P. Coullet, C. Riera, and C. Tresser. Stable static localized structures in one dimension. Phys. Rev. Lett., 84:3069-3072, Apr 2000.

[14] P. Coullet, C. Riera, and C. Tresser. A new approach to data storage using localized structures. Chaos, 14:193201, Mar 2004.

[15] John Mallet-Paret and Roger D. Nussbaum. Global continuation and asymptotic behaviour for periodic solutions of a differential-delay equation. Comput. Math. Math. Phys., 38(3):457-465, 1998.

[16] Michel Nizette. Front dynamics in a delayed-feedback system with external forcing. Physica D: Nonlinear Phenomena, 183(3-4):220 - 244, 2003.

[17] F. Leo, S. Coen, P. Kockaert, S.P. Gorza, P. Emplit, and M. Haelterman. Temporal cavity solitons in onedimensional kerr media as bits in an all-optical buffer. Nat Photon, 4(7):471-476, Jul 2010.

[18] Mustapha Tlidi and Lendert Gelens. High-order dispersion stabilizes dark dissipative solitons in all-fiber cavities. Opt. Lett., 35(3):306-308, Feb 2010.

[19] M. Tlidi, L. Bahloul, L. Cherbi, A. Hariz, and S. Coulibaly. Drift of dark cavity solitons in a photoniccrystal fiber resonator. Phys. Rev. A, 88:035802, Sep 2013.

[20] T. Herr, V. Brasch, J. D. Jost, C. Y. Wang, N. M. Kondratiev, M. L. Gorodetsky, and T. J. Kippenberg. Temporal solitons in optical microresonators. Nature Photonics, 8(2):145-152, 2014.

[21] M. Marconi, J. Javaloyes, S. Balle, and M. Giudici. How lasing localized structures evolve out of passive mode locking. Phys. Rev. Lett., 112:223901, Jun 2014.

[22] M. Marconi, J. Javaloyes, S. Balle, and M. Giudici. Passive mode-locking and tilted waves in broad-area vertical cavity surface emitting lasers. Selected Topics in Quantum Electronics, IEEE Journal of, PP(99):1-1, 2014.

[23] B. Garbin, J. Javaloyes, G. Tissoni, and S. Barland. Topological solitons as addressable phase bits in a driven laser. Nat. Com., 6, 2015.

[24] N. N. Rosanov and G. V. Khodova. Autosolitons in bistable interferometers. Opt. Spectrosc., 65:449, 1988.

[25] M. Tlidi, P. Mandel, and R. Lefever. Localized structures and localized patterns in optical bistability. Phys. Rev. Lett., 73:640-643, Aug 1994.

[26] W. J. Firth and A. J. Scroggie. Optical bullet holes: Robust controllable localized states of a nonlinear cavity. Phys. Rev. Lett., 76:1623-1626, Mar 1996.

[27] S. Barland, J. R. Tredicce, M. Brambilla, L. A. Lugiato, 
S. Balle, M. Giudici, T. Maggipinto, L. Spinelli, G. Tissoni, T. Knödl, M. Miller, and R. Jäger. Cavity solitons as pixels in semiconductor microcavities. Nature, 419(6908):699-702, Oct 2002.

[28] P. Genevet, S. Barland, M. Giudici, and J. R. Tredicce. Cavity soliton laser based on mutually coupled semiconductor microresonators. Phys. Rev. Lett., 101:123905, Sep 2008.

[29] Y. Tanguy, T. Ackemann, W. J. Firth, and R. Jäger. Realization of a semiconductor-based cavity soliton laser. Phys. Rev. Lett., 100:013907, Jan 2008.

[30] F. Pedaci, G. Tissoni, S. Barland, M. Giudici, and J. R. Tredicce. Mapping local defects of extended media using localized structures. Appl. Phys. Lett., 93:111104, 2008.

[31] T. Ackemann, N. Radwell, Y. Noblet, and R. Jäger. Disorder mapping in VCSELs using frequency-selective feedback. Opt. Lett., 37:1079-1081, 2012.

[32] F. T. Arecchi, G. Giacomelli, A. Lapucci, and R. Meucci. Two-dimensional representation of a delayed dynamical system. Phys. Rev. A, 45:R4225-R4228, Apr 1992.

[33] G. Giacomelli and A. Politi. Relationship between delayed and spatially extended dynamical systems. Phys. Rev. Lett., 76:2686-2689, Apr 1996.

[34] S.A. Kashchenko. The Ginzburg-Landau equation as a normal form for a second-order difference-differential equation with a large delay. Comput. Math. Math. Phys., 38(3):1, 1998.

[35] Giovanni Giacomelli, Francesco Marino, Michael A. Zaks, and Serhiy Yanchuk. Coarsening in a bistable system with long-delayed feedback. EPL (Europhysics Letters), 99(5):58005, 2012.

[36] Giovanni Giacomelli, Francesco Marino, Michael A. Zaks, and Serhiy Yanchuk. Nucleation in bistable dynamical systems with long delay. Phys. Rev. E, 88:062920, Dec 2013.

[37] Francesco Marino, Giovanni Giacomelli, and Stephane
Barland. Front pinning and localized states analogues in long-delayed bistable systems. Phys. Rev. Lett., 112:103901, Mar 2014.

[38] Michel Nizette. Stability of square oscillations in a delayed-feedback system. Phys. Rev. E, 70:056204, Nov 2004 .

[39] Athanasios Gavrielides, Thomas Erneux, David W. Sukow, Guinevere Burner, Taylor McLachlan, John Miller, and Jake Amonette. Square-wave self-modulation in diode lasers with polarization-rotated optical feedback. Opt. Lett., 31(13):2006-2008, Jul 2006.

[40] J. Mulet, M. Giudici, J. Javaloyes, and S. Balle. Squarewave switching by crossed-polarization gain modulation in vertical-cavity semiconductor lasers. Phys. Rev. A, 76:043801, Oct 2007.

[41] Lionel Weicker, Thomas Erneux, Otti D'Huys, Jan Danckaert, Maxime Jacquot, Yanne Chembo, and Laurent Larger. Strongly asymmetric square waves in a timedelayed system. Phys. Rev. E, 86:055201, Nov 2012.

[42] Serhiy Yanchuk, Leonhard Lücken, Matthias Wolfrum, and Alexander Mielke. Spectrum and amplitude equations for scalar delay-differential equations with large delay. Discrete and Continuous Dynamical Systems, 35(1):537-553, 2015.

[43] P. Coullet. Localized patterns and fronts in nonequilibrium systems. International Journal of Bifurcation and Chaos, 12(11):2445-2457, 2002.

[44] Matthias Wolfrum and Serhiy Yanchuk. Eckhaus instability in systems with large delay. Physical review letters, 96(22):220201, 2006.

[45] Michel Nizette, Thomas Erneux, Athanasios Gavrielides, and Vassilios Kovanis. Averaged equations for injection locked semiconductor lasers. Physica D: Nonlinear Phenomena, 161(3-4):220 - 236, 2002. 